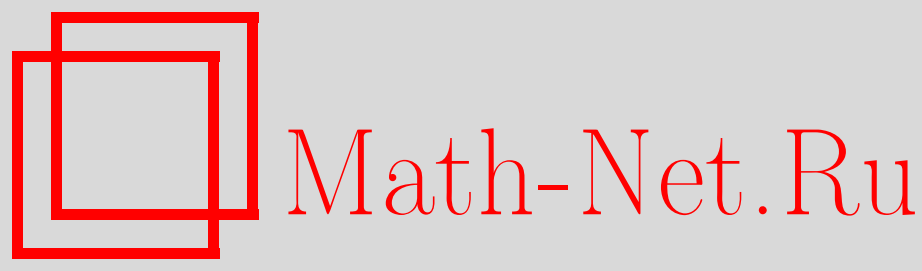

В. В. Дубс, В. В. Прудников, П. В. Прудников, Ренормгрупповое описание влияния дефектов структуры на фазовый переход в сложных спиновых системах с эффектами случайной анизотропии и дефектами структуры, TMФ, 2017, том 190, номер 3, 419-425

DOI: https://doi.org/10.4213/tmf9113

Использование Общероссийского математического портала Math-Net.Ru подразумевает, что вы прочитали и согласны с пользовательским соглашением http://www . mathnet.ru/rus/agreement

Параметры загрузки:

IP : 3.95 .254 .165

26 апреля 2023 г., 08:22:42

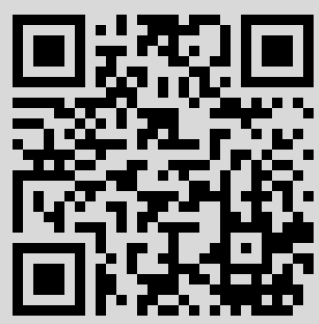




\title{
ФИЗИКА
}

Том 190, № 3

март, 2017

(C) 2017 г. $\quad$ В. В. Дубс*, В. В. Прудников*, П. В. Прудников*

\section{РЕНОРМГРУППОВОЕ ОПИСАНИЕ ВЛИЯНИЯ ДЕФЕКТОВ СТРУКТУРЫ НА ФАЗОВЫЙ ПЕРЕХОД В СЛОЖНЫХ СПИНОВЫХ СИСТЕМАХ С ЭФФЕКТАМИ СЛУЧАЙНОЙ АНИЗОТРОПИИ И ДЕФЕКТАМИ СТРУКТУРЫ}

\begin{abstract}
Впервые осуществлено теоретико-полевое описание фазового перехода в аморфном магнетике с эффектами случайной анизотропии и влиянием дефектов структуры в рамках двухпетлевого приближения при фиксированной размерности $d=3$. Для данной многовершинной модели определена система неподвижных точек ренормгрупповых уравнений и проведен расчет показателей их устойчивости с применением метода суммирования Паде-Бореля. Показана роль дефектов структуры как стабилизирующих факторов при реализации фазовых переходов второго рода.
\end{abstract}

Ключевые слова: фазовый переход, аморфные магнетики, анизотропия, дефекты структуры, метод ренормгруппы, методы суммирования.

DOI: https://doi.org/10.4213/tmf9113

Известно, что критерии теории Ландау реализации фазового перехода второго рода являются необходимыми условиями их осуществления, однако вблизи критической температуры аномально большие флуктуации могут как сохранить фазовый переход второго рода, так и привести к флуктуационному срыву к фазовому переходу первого рода. Определить тип фазового перехода в таком случае помогает исследование устойчивости набора неподвижных точек ренормгрупповых уравнений системы [1]-[4]. В ренормгрупповой теории выявлен ряд систем с многокомпонентным параметром порядка, в которых осуществляется флуктуационный срыв к фазовым переходам первого рода [5]-[7].

Исследование выполнено за счет гранта Российского научного фонда (проект № 14-12-00562).

* Омский государственный университет им. Ф. М. Достоевского, Омск, Россия. E-mail: prudnikv@mail.ru, soranoo@mail.ru 
Исследования методом Монте-Карло систем, описываемых моделью Поттса и содержащих дефекты структуры в виде немагнитных примесей, указывают на существование возможности стабилизации фазового перехода второго рода посредством внедрения в систему дефектов [8]. Кроме того, в работе [9] было показано, что эффекты случайной анизотропии могут привести к усилению стабильности фазового перехода второго рода из-за усложнения симметрии системы.

Таким образом, с точки зрения исследования влияния флуктуаций на устойчивость фазовых переходов второго рода большой интерес представляет изучение анизотропных магнитных систем, содержащих немагнитные примеси. Ренормгрупповое описание такой системы в рамках применения однопетлевого приближения и метода $\varepsilon$-разложения осуществлено в работе [10]. Авторы высказали предположение о существовании двух областей в фазовом пространстве параметров гамильтониана системы, соответствующих двум различным сценариям осуществления фазового перехода. Для параметров, принадлежащих первой области, гамильтониан задачи под действием ренормгрупповых потоков переходит в неустойчивое состояние, соответствующее фазовому переходу первого рода, а в случае принадлежности ко второй области ренормгрупповые потоки переводят гамильтониан в устойчивую неподвижную точку, соответствующую фазовому переходу второго рода. Для построения более точного описания необходимо провести анализ ренормгрупповых уравнений системы в рамках двухпетлевого приближения при фиксированной размерности $d=3$, применяя к рядам теории методы суммирования асимптотических рядов.

Рассмотрим фазовый переход в аморфных магнетиках с эффектами случайной анизотропии. Случай равномерного распределения эффектов анизотропии характеризуется гамильтонианом $H_{\mathrm{e}}[9]$ :

$$
\begin{aligned}
H_{\mathrm{e}}= & \int d^{d} R\left(\frac{1}{2}\left[\mu_{0}^{2}|\vec{\varphi}|^{2}+|\vec{\nabla} \vec{\varphi}|^{2}\right]+u_{0}|\vec{\varphi}|^{4}+v_{0} \sum_{\alpha=1}^{n}\left|\overrightarrow{\varphi_{\alpha}}\right|^{4}+\right. \\
& \left.+w_{0} \sum_{i, j}^{m} \sum_{\alpha, \beta=1}^{n} \varphi_{i}^{\alpha} \varphi_{j}^{\alpha} \varphi_{i}^{\beta} \varphi_{j}^{\beta}\right) .
\end{aligned}
$$

Случаю кубического распределения эффектов анизотропии соответствует гамильтониан $H_{\mathrm{c}}[9]$ :

$$
\begin{aligned}
H_{\mathrm{c}}= & \int d^{d} R\left(\frac{1}{2}\left[\mu_{0}^{2}|\vec{\varphi}|^{2}+|\vec{\nabla} \vec{\varphi}|^{2}\right]+u_{0}|\vec{\varphi}|^{4}+v_{0} \sum_{\alpha=1}^{n}\left|\overrightarrow{\varphi_{\alpha}}\right|^{4}+\right. \\
& \left.+w_{0} \sum_{i=1}^{m} \sum_{\alpha, \beta=1}^{n}\left(\varphi_{i}^{\alpha}\right)^{2}\left(\varphi_{i}^{\beta}\right)^{2}+y_{0} \sum_{i=1}^{m} \sum_{\alpha=1}^{n}\left(\varphi_{i}^{\alpha}\right)^{4}\right) .
\end{aligned}
$$

В рассматриваемом нами случае фазового перехода в аморфных магнетиках с эффектами случайной анизотропии, содержащих дефекты структуры, система характеризуется четырехкомпонентным параметром порядка. Соответствующий гамильтониан состоит из членов $H_{\mathrm{e}}$ и $H_{\mathrm{c}}$ и имеет следующий вид [10]:

$$
\begin{aligned}
H= & \int d^{d} R\left(\frac{1}{2}\left[\mu_{0}^{2}|\vec{\varphi}|^{2}+|\vec{\nabla} \vec{\varphi}|^{2}\right]+u_{0}|\vec{\varphi}|^{4}+v_{0} \sum_{\alpha=1}^{n}\left|\overrightarrow{\varphi_{\alpha}}\right|^{4}+\right. \\
& \left.+w_{0} \sum_{i=1}^{m} \sum_{\alpha, \beta=1}^{n}\left(\varphi_{i}^{\alpha}\right)^{2}\left(\varphi_{i}^{\beta}\right)^{2}+y_{0} \sum_{i=1}^{m} \sum_{\alpha=1}^{n}\left(\varphi_{i}^{\alpha}\right)^{4}+z_{0} \sum_{i, j}^{m} \sum_{\alpha, \beta=1}^{n} \varphi_{i}^{\alpha} \varphi_{j}^{\alpha} \varphi_{i}^{\beta} \varphi_{j}^{\beta}\right),
\end{aligned}
$$


где затравочные значения вершин характеризуются условиями $u_{0}>0, v_{0}>0$, $w_{0}<0$. Здесь вершина $u$ является полимерной, $v$ - изотропной, $y$ - кубической, а вершины $w, z$ отвечают за эффекты случайной анизотропии и влияние дефектов структуры.

Система реногмгрупповых уравнений, полученная в репличном пределе $n \rightarrow 0$ в рамках двухпетлевого приближения непосредственно при $d=3$, характеризуется приведенным ниже набором $\beta$-функций для перенормированных вершин, а также $\gamma_{\varphi^{-}}, \gamma_{\varphi^{2}}$-функциями, позволяющими определять критические индексы [3], [4]:

$$
\begin{aligned}
\beta_{u}= & -\left\{u-\frac{1}{6}\left[8 u^{2}+2(m+2) u v+2 v w+4 u w+6 u y+2(m+1) u z+2 v z+\right.\right. \\
& \left.+2 w z+3 z^{2}\right]+\frac{1}{9}\left[44 u^{3}+48 u^{2} w+12 w^{2} u+24(m+2) v u^{2}+6(m+2) u v^{2}+\right. \\
& +4 v^{2} w+4 w^{2} v+72 u^{2} y+18 y^{2} u+60 u v w+36 u v y+36 u w y+28 z^{3}+26 u z^{2}+ \\
& +56 u^{2} z+12(m+2) v z^{2}+3(m+2) v^{2} z+20 y z^{2}+28 w z^{2}+4 w^{2} z+28 u w z+ \\
& +20 u y z+20 v w z+6 v y z+20 w y z+6(m+2) u v z] i_{1}+ \\
& +\frac{2}{9}\left[2 u^{3}+2(m+2) v u^{2}+(m+2) u v^{2}+2 w^{2} u+6 u w v+4 u^{2} w+6 u^{2} y+6 u v y+\right. \\
& \left.\left.+6 u w y+3 y^{2} u+6 z u y+6 u z w+2(m+2) z u^{2}(m+2) u z v+8(m+2) u z^{2}\right] i_{2}\right\}
\end{aligned}
$$

$$
\begin{aligned}
\beta_{v}= & -\left\{v-\frac{1}{6}\left[(m+8) v^{2}+12 v u+4 v w+6 v y+6 y z+2(m+5) v z\right]+\right. \\
& +\frac{1}{9}\left[2(5 m+22) v^{3}+24(m+5) v^{2} u+84 v u^{2}+12 v w^{2}+68 v^{2} w+72 v^{2} y+\right. \\
& +18 y^{2} v+72 u v w+108 u v y+36 w v y+(5 m+22) v z^{2}+(5 m+22) v^{2} z+36 v w y+ \\
& \left.+48 y z^{2}+36 y^{2} z+4(m+26) v u z+48 v w z+72 v y z+48 y z u+24 y z w\right] i_{1}+ \\
& +\frac{2}{9}\left[(m+2) v^{3}+2(m+2) u v^{2}+2 v u^{2}+2 v w^{2}+6 v^{2} w+4 v u w+6 v u y+6 v^{2} y+\right. \\
& \left.\left.+6 v w y+3 v y^{2}+6 v z y+5 v z w+2(m+2) v z u+(m+2) z v^{2}+8(m+2) v z^{2}\right] i_{2}\right\},
\end{aligned}
$$

$$
\begin{aligned}
\beta_{w}= & -\left\{w-\frac{1}{6}\left[8 w^{2}+122 u w+42 v w+62 y w+10 z w+6 y z\right]+\right. \\
& +\frac{1}{9}\left[44 w^{3}+84 u^{2} w+120 w^{2} u+2(m+6) w v^{2}+68 v w^{2}+72 w^{2} y+18 w y^{2}+\right. \\
& +12(m+6) w u v+108 w u y+36 w v y+(m+6) w z^{2}+44 w^{2} z+18 z y^{2}+20 z^{2} y+ \\
& +28 w u z+(m+6) w v z+20 w y z+32 y z u+6 y z v] i_{1}+ \\
& +\frac{2}{9}\left[(m+2) w v^{2}+2(m+2) w u v+2 w u^{2}+2 w^{3}+6 v w^{2}+4 u w^{2}+6 u y w+6 v y w+\right. \\
& \left.\left.+6 w^{2} y+3 w y^{2}+w z y+6 z w^{2}+2(m+2) w z u+(m+2) w z v+8(m+2) w z^{2}\right] i_{2}\right\},
\end{aligned}
$$




$$
\begin{aligned}
& \beta_{y}=-\left\{y-\frac{1}{6}\left[9 y^{2}+8 v w+12 u y+12 v y+12 w y+6 y z\right]+\right. \\
& +\frac{1}{9}\left[4(m+18) v^{2} w+72 w^{2} v+54 y^{3}+84 u^{2} y+6(m+14) v^{2} y+84 w^{2} y+\right. \\
& +144 y^{2} u+144 y^{2} v+144 y^{2} w+96 u v w+12(m+14) v^{2} y+252 v w y+168 u w y+ \\
& \left.+24 y z^{2}+72 y^{2} z+24 y u z+36 y v z+48 y w z+24 v w z\right] i_{1}+ \\
& +\frac{2}{9}\left[2 u^{2} y+2(m+2) u v y+(m+2) v^{2} y+6 v w y+2 w^{2} y+4 u w y+6 y^{2} u+6 y^{2} v+\right. \\
& \left.\left.+6 y^{2} w+3 y^{3}+6 z y^{2}+6 z w y+2(m+2) z u y+(m+2) z v y+8(m+2) z^{2} y\right] i_{2}\right\} \\
& \beta_{z}=-\left\{z-\frac{1}{6}\left[(m+4) z^{2}+12 z u+4 z w+4 z v\right]+\right. \\
& +\frac{1}{9}\left[(5 m+22) z^{3}+28 z u^{2}+6(m+5) z^{2} u+6(m+5) z v^{2}+40 z^{2} v+12 z w^{2}+\right. \\
& +68 z^{2} w+36 z^{2} y+36 z u w+36 z v w+108 z u y+12(m+5) z u v+36 z w y+ \\
& +6 z y v] i_{1}+\frac{2}{9}\left[2 u^{2} z+2(m+2) u v z+(m+2) v^{2} z+6 v w z+2 w^{2} z+4 u w z+\right. \\
& \left.\left.+6 u y z+6 v y z+6 w y z+3 y^{2} z+6 z^{2} y+6 z^{2} w+(m+2) z^{2} v+8(m+2) z^{3}\right] i_{2}\right\}, \\
& \gamma_{\varphi}=-\frac{1}{9}\left(2 u^{2}+2(m+2) u v+(m+2) v^{3}+6 v w+2 w^{2}+4 v w+6 u y+6 v y+\right. \\
& \left.+6 w y+3 y^{2}+6 z y+6 z w+2(m+2) z u+(m+2) z v+8(m+2) z^{2}\right) i_{2}, \\
& \gamma_{\varphi^{2}}=\frac{1}{3}\left(\frac{1}{2}[2 u+(m+2) v+2 w+y+(m+2) z]-\left[w u^{2}+2(m+2) u v+(m+2) v^{2}+\right.\right. \\
& +6 v w+2 w^{2}+4 u w+6 u y+6 v y+6 w y+3 y^{2}+2(m+2) z u+(m+2) z v+ \\
& \left.\left.+6 z w+6 z y+2(m+2) z^{2}\right] i_{1}\right)
\end{aligned}
$$

где факторы $i_{1}$ и $i_{2}$, учитывающие вклады от двухпетлевых диаграмм, характеризуются следующими выражениями [11]:

$$
\begin{gathered}
i_{1}=\frac{D_{2}}{J_{1}^{2}}-\frac{1}{2}, \quad i_{2}=-\frac{D_{1}^{\prime}}{J_{1}^{2}}, \quad J_{1}=\int \frac{d q}{\left(q^{2}+1\right)^{2}}=(4 \pi)^{-d / 2} \Gamma\left(2-\frac{1}{2} d\right), \\
D_{1}^{\prime}=\left.\frac{\delta}{\delta k^{2}} \int \frac{d q_{1} d q_{2}}{\left(q_{1}^{2}+1\right)^{2}\left(q_{2}^{2}+1\right)^{2}} \frac{1}{\left(q_{1}+q_{2}+k\right)^{2}+1}\right|_{k=0}, \\
D_{2}=\int \frac{d q_{1} d q_{2}}{\left(q_{1}^{2}+1\right)^{2}\left(q_{2}^{2}+1\right)^{2}} \frac{1}{\left(q_{1}+q_{2}\right)^{2}+1} .
\end{gathered}
$$

При $d=3$ верны равенства $i_{1}=1 / 6, i_{2}=-2 / 27$. 
Неподвижные точки ренормгрупповых уравнений определяются нулями $\beta$-функций. Мы провели их поиск с помощью метода суммирования Паде-Бореля, адаптированного на случай многопараметрических рядов посредством применения $\lambda$-метода [3], [4]. Проведенная процедура позволила выявить неподвижные точки для соответствующих значений компонент $m$ параметра порядка. Значения вершин в неподвижных точках приведены в табл. 1.

ТАБлицА 1. Значения вершин в неподвижных точках, полученные после процедуры суммирования.

\begin{tabular}{|c|c|c|c|c|c|c|}
\hline$\Phi . \mathrm{T}$. & $m$ & $u^{*}$ & $v^{*}$ & $w^{*}$ & $y^{*}$ & $z^{*}$ \\
\hline I & $\forall$ & 0 & 0 & 0 & 0 & 0 \\
\hline II & $\begin{array}{l}2 \\
3 \\
4\end{array}$ & $\begin{array}{l}0 \\
0 \\
0\end{array}$ & $\begin{array}{l}0.9107 \\
0.8102 \\
0.7275\end{array}$ & $\begin{array}{l}0 \\
0 \\
0\end{array}$ & $\begin{array}{l}0 \\
0 \\
0\end{array}$ & $\begin{array}{l}0 \\
0 \\
0\end{array}$ \\
\hline III & $\forall$ & 1.1857 & 0 & 0 & 0 & 0 \\
\hline IV & $\forall$ & 0 & 0 & 0 & 1.0339 & 0 \\
\hline $\mathrm{V}$ & $\begin{array}{l}3 \\
4 \\
\end{array}$ & $\begin{array}{l}0.1733 \\
0.2867 \\
\end{array}$ & $\begin{array}{l}0.6460 \\
0.4851 \\
\end{array}$ & $\begin{array}{l}0 \\
0 \\
\end{array}$ & $\begin{array}{l}0 \\
0 \\
\end{array}$ & $\begin{array}{l}0 \\
0 \\
\end{array}$ \\
\hline VI & $\forall$ & 2.1112 & 0 & -2.1112 & 0 & 0 \\
\hline VII & $\begin{array}{l}2 \\
3 \\
4 \\
\end{array}$ & $\begin{array}{l}0 \\
0 \\
0\end{array}$ & $\begin{array}{l}1.5507 \\
0.8293 \\
0.5259 \\
\end{array}$ & $\begin{array}{l}0 \\
0 \\
0\end{array}$ & $\begin{array}{r}-1.0340 \\
-0.0585 \\
0.3624 \\
\end{array}$ & $\begin{array}{l}0 \\
0 \\
0\end{array}$ \\
\hline VIII & $\begin{array}{l}3 \\
4\end{array}$ & $\begin{array}{l}0.1695 \\
0.2751\end{array}$ & $\begin{array}{l}0.7096 \\
0.4196\end{array}$ & $\begin{array}{l}0 \\
0\end{array}$ & $\begin{array}{r}-0.1022 \\
0.1432\end{array}$ & $\begin{array}{l}0 \\
0\end{array}$ \\
\hline IX & $\forall$ & 0.6677 & 0 & -0.6677 & 1.0339 & 0 \\
\hline $\mathrm{X}$ & $\forall$ & 0 & 0 & -0.4401 & 1.5932 & 0 \\
\hline $\mathrm{XI}$ & $\begin{array}{l}2 \\
3 \\
4 \\
\end{array}$ & $\begin{array}{l}0 \\
0 \\
0\end{array}$ & $\begin{array}{l}1.7132 \\
0.4171 \\
0.3971 \\
\end{array}$ & $\begin{array}{l}0 \\
0 \\
0\end{array}$ & $\begin{array}{r}-0.5446 \\
-0.1338 \\
0.2542\end{array}$ & $\begin{array}{l}-0.5911 \\
-0.3379 \\
-0.5473\end{array}$ \\
\hline XII & $\begin{array}{l}3 \\
4\end{array}$ & $\begin{array}{l}0.6288 \\
0.1306\end{array}$ & $\begin{array}{l}0 \\
0\end{array}$ & $\begin{array}{l}0 \\
0\end{array}$ & $\begin{array}{l}1.1021 \\
1.5761\end{array}$ & $\begin{array}{l}-0.4073 \\
-0.2181\end{array}$ \\
\hline XIII & $\begin{array}{l}2 \\
3 \\
4 \\
\end{array}$ & $\begin{array}{l}0.6564 \\
0.7031 \\
0.7462 \\
\end{array}$ & $\begin{array}{l}0.8135 \\
0.6073 \\
0.4391 \\
\end{array}$ & $\begin{array}{l}0 \\
0 \\
0\end{array}$ & $\begin{array}{l}0 \\
0 \\
0\end{array}$ & $\begin{array}{l}-0.3313 \\
-0.4233 \\
-0.2237\end{array}$ \\
\hline XIV & $\begin{array}{l}3 \\
4\end{array}$ & $\begin{array}{l}1.5179 \\
1.3995\end{array}$ & $\begin{array}{l}0 \\
0\end{array}$ & $\begin{array}{l}0 \\
0\end{array}$ & $\begin{array}{l}0 \\
0\end{array}$ & $\begin{array}{l}-1.3014 \\
-0.9482\end{array}$ \\
\hline
\end{tabular}

Первые десять неподвижных точек в этой таблице соответствуют системе без дефектов структуры, так как они характеризуются равенством $z^{*}=0$. Эта система исследовалась в работе [9], в которой было показано, что неподвижная точка Х является устойчивой и физически достижимой, т. е. ей соответствует фазовый переход второго рода. Неподвижные точки XI-XIV описывают влияние дефектов структуры.

Для исследования устойчивости неподвижных точек необходимо линеаризовать систему уравнений ренормгруппы (4)-(8). Результат линеаризации в матричной 
форме имеет вид

$$
\left[\begin{array}{c}
\Delta u^{\prime} \\
\Delta v^{\prime} \\
\Delta w^{\prime} \\
\Delta y^{\prime} \\
\Delta z^{\prime}
\end{array}\right]=M\left[\begin{array}{c}
\Delta u \\
\Delta v \\
\Delta w \\
\Delta y \\
\Delta z
\end{array}\right]
$$

где $\Delta u=u-u^{*}, \Delta v=v-v^{*}, \Delta w=w-w^{*}, \Delta y=y-y^{*}, \Delta z=z-z^{*}$. Величины с символом * характеризуют значения вершин в неподвижных точках. Для того чтобы сделать вывод о характере устойчивости неподвижной точки [3], [4], мы ввели матрицу $M$, выражающуюся через производные от $\beta$-функций:

$$
M=\left[\begin{array}{lllll}
\frac{\delta \beta_{u}}{\delta u} & \frac{\delta \beta_{u}}{\delta v} & \frac{\delta \beta_{u}}{\delta w} & \frac{\delta \beta_{u}}{\delta y} & \frac{\delta \beta_{u}}{\delta z} \\
\frac{\delta \beta_{v}}{\delta u} & \frac{\delta \beta_{v}}{\delta v} & \frac{\delta \beta_{v}}{\delta w} & \frac{\delta \beta_{v}}{\delta y} & \frac{\delta \beta_{v}}{\delta z} \\
\frac{\delta \beta_{w}}{\delta u} & \frac{\delta \beta_{w}}{\delta v} & \frac{\delta \beta_{w}}{\delta w} & \frac{\delta \beta_{w}}{\delta v} & \frac{\delta \beta_{w}}{\delta z} \\
\frac{\delta \beta_{y}}{\delta u} & \frac{\delta \beta_{y}}{\delta v} & \frac{\delta \beta_{y}}{\delta w} & \frac{\delta \beta_{y}}{\delta y} & \frac{\delta \beta_{y}}{\delta z} \\
\frac{\delta \beta_{z}}{\delta u} & \frac{\delta \beta_{z}}{\delta v} & \frac{\delta \beta_{z}}{\delta w} & \frac{\delta \beta_{z}}{\delta y} & \frac{\delta \beta_{z}}{\delta z}
\end{array}\right],
$$

которую нужно привести к диагональному виду

$$
M=\left[\begin{array}{ccccc}
e^{-\lambda_{u}} & 0 & 0 & 0 & 0 \\
0 & e^{-\lambda_{v}} & 0 & 0 & 0 \\
0 & 0 & e^{-\lambda_{w}} & 0 & 0 \\
0 & 0 & 0 & e^{-\lambda_{y}} & 0 \\
0 & 0 & 0 & 0 & e^{-\lambda_{z}}
\end{array}\right]
$$

где $\lambda_{u}, \lambda_{v}, \lambda_{w}, \lambda_{y}, \lambda_{z}-$ показатели матрицы устойчивости.

Если все показатели $\lambda_{i}$ матрицы устойчивости, вычисленные в соответствующей неподвижной точке, принимают отрицательные значения, то неподвижная точка является устойчивой. Если хотя бы один из показателей $\lambda_{i}$ положителен, то неподвижная точка является неустойчивой.

Проведенное нами исследование устойчивости полученных неподвижных точек показало, что за счет влияния дефектов структуры, характеризующегося вершиной $z$, неподвижная точка $\mathrm{X}$ теряет устойчивость, а приобретает устойчивость неподвижная примесная точка XI для $m=3$ (случай кубической анизотропии). Следовательно, для данного случая присутствие дефектов структуры становится стабилизирующим фактором для реализации фазового перехода второго рода в системе. Однако дефекты структуры одновременно оказывают дестабилизирующее воздействие на неподвижные точки, характеризующие эффекты случайной анизотропии.

В устойчивой неподвижной точке XI для $m=3$ можно вычислить критические индексы $\eta$ и $\nu$, определяемые следующими соотношениями:

$$
\begin{gathered}
\eta=\gamma_{\varphi}\left(u^{*}, v^{*}, w^{*}, y^{*}, z^{*}\right), \\
\nu=\left[2+\gamma_{\varphi^{2}}\left(u^{*}, v^{*}, w^{*}, y^{*}, z^{*}\right)-\gamma_{\varphi}\left(u^{*}, v^{*}, w^{*}, y^{*}, z^{*}\right)\right]^{-1} .
\end{gathered}
$$


Поскольку правые части этих выражений определены в виде асимптотических рядов, для их вычисления также был применен обобщенный на многопараметрический случай метод суммирования Паде-Бореля. В результате были получены следующие значения критических индексов:

$$
\eta=0.026(3), \quad \nu=0.678(4) .
$$

Значения остальных статических критических индексов для аморфных магнетиков с эффектами случайной кубической анизотропии и влиянием дефектов структуры можно получить на основе значений критических индексов $\eta$ и $\nu$ при использовании скейлинговых соотношений [1], [2], связывающих критические индексы между собой.

В результате проведенных исследований на примере многовершинной модели аморфного магнетика мы показали стабилизирующую роль дефектов структуры при реализации фазового перехода второго рода в системе с многокомпонентным параметром порядка.

Благодарности. Авторы благодарят организаторов V международной конференции "Модели квантовой теории поля", посвященной памяти А. Н. Васильева, за гостеприимство и высокий уровень организации.

\section{Список литературы}

[1] К. Вильсон, Дж. Когут, Ренормализационная группа и в-разложение, Мир, М., 1975.

[2] D. J. Amit, Field Theory the Renormalization Group and Critical Phenomena, McGrawHill, New York, 1978.

[3] В. В. Прудников, П.В. Прудников, А.Н. Вакилов, Теоретико-полевые и численные методы описания критических явлений в структурно неупорядоченных системах, Изд-во Омск. гос. ун-та, Омск, 2012.

[4] В. В. Прудников, П. В. Прудников, А. Н. Вакилов, Теоретические методы описания неравновесного критического поведения структурно неупорядоченных систем, Физматлит, М., 2013.

[5] И. Ф. Люксютов, В. Л. Покровский, “Фазовые переходы второго рода в системах с кубической анизотропией”, Писъма в ЖЭТФ, 21:1 (1975), 22-25.

[6] D. Mukamel, S. Krinsky, "Physical realizations of $n \geqslant 4$-component vector models. I. Derivation of the Landau-Ginzburg-Wilson Hamiltonians", Phys. Rev. B., 13:11 (1976), 5065-5077.

[7] D. Mukamel, S. Krinsky, "Physical realizations of $n \geqslant 4$-component vector models. II. E-expansion analysis of the critical behavior", Phys. Rev. B., 13:11 (1976), 5078-5085.

[8] А.К. Муртазаев, Ф.Б. Бабаев, Г.Я. Азнаурова, "Особенности фазовых переходов в трехмерных разбавленных структурах, описываемых моделью Поттса", ЖЖЭТФ, 136:3 (2009), 516-520.

[9] M. Dudka, R. Folk, Yu. Holovatch, "On the critical behaviour of random anisotropy magnets: cubic anisotropy", Condens. Matter Phys., 4:3(27) (2001), 459-472.

[10] D. Mukamel, G. Grinstein, "Critical behavior of random systems", Phys. Rev. B., 25:1 (1982), 381-388.

[11] G. Jug, "Critical behavior of disordered spin systems in two and three dimensions", Phys. Rev. B., 27:1 (1983), 609-612. 